\title{
Traveling-Wave Tube Amplifier Model to Predict High-Order Modulation Intersymbol Interference
}

\author{
Carol L. Kory ${ }^{1}$ and Monty Andro ${ }^{2}$ \\ ${ }^{1}$ Analex Corporation $/ 2$ NASA Glenn Research Center \\ 21000 Brookpark Road, MS 54-5 \\ Cleveland, $\mathrm{OH} 44135$ \\ 216-433-3512 \\ Carol.L.Kory@grc.nasa.gov
}

Demands for increased data rates in satellite communications necessitate higher order modulation schemes, larger system bandwidth and minimum distortion of the modulated signal as it is passed through the traveling wave tube amplifier (TWTA). One type of distortion that the TWTA contributes to is intersymbol interference (ISI), and this becomes particularly disruptive with wide-band, complex modulation schemes. It is suspected that in addition to the dispersion of the TWT, frequency dependent reflections due to mismatches within the TWT are a significant contributor to ISI. To experimentally investigate the effect of these mismatches within the physical TWT on ISI would be prohibitively expensive, as it would require manufacturing numerous amplifiers in addition to the acquisition of the required digital hardware.

Conventionally, effects on digital data from the TWTA are investigated using communication system level software. These models use a nonlinear, black-box model to represent the TWTA. Currently, SPW [1] is used in-house at NASA Glenn Research Center (GRC), where the behavior of the TWTA is characterized by a memoryless envelope model. The model parameters are obtained from the AM/AM (output power versus input power) and AM/PM (output phase versus input power) conversions, which are typically obtained by sweeping the input amplitude at the center frequency either experimentally or by using conventional frequency domain TWT codes [2]. Thus, the TWT characteristics are assumed constant (memoryless) over the bandwidth of the simulated signal. In addition, the model does not account for the memory effects of signal reflections occurring within the TWT. As data rate requirements increase, thus requiring complex, high-order modulation, and wide-band digital signals, these assumptions become less accurate.

In an attempt to develop a more accurate model to correlate ISI with the TWTA and the operational signal, a fully three-dimensional (3D), time-dependent, TWT interaction model has been developed using the electromagnetic particle-in-cell (PIC) code MAFIA (Solution of MAxwell's equations by the FiniteIntegration-Algorithm). The model includes a user defined slow-wave circuit with a spatially tapered region of loss to implement a sever, and spatially varied geometry (such as helical pitch) to implement a phase velocity taper. The model also includes user defined input/output coupling and an electron beam contained by solenoidal, electrostatic or periodic permanent magnet (PPM) focusing allowing standard or novel TWT's to be investigated. This model comprehensively takes into account the effects of frequency dependent nonlinear distortions (AM/AM and AM/PM); gain ripple due to frequency dependent reflections at the input/output coupling, severs, and mismatches from dynamic pitch variations; drive induced oscillations; harmonic generation; intermodulation products; and backward waves. The user is able to define any excitation function so that digitally modulated signals can be used as input and computational correlation of ISI with TWT parameters can be conducted. This type of system level nonlinear model can be expected to possess superior predictive fidelity compared to models based on swept-tone, swept-amplitude data. The latter data is partial because superposition does not apply in the nonlinear device, and the interactions between frequency components in a realistic signal are not captured $[5]$.

This is a preprint or reprint of a paper intended for presentation at a conference. Because changes may be made before formal publication, this is made available with the understanding that it will not be cited or reproduced without the permission of the author. 
The MAFIA TWT interaction model will be described, and results will be presented where this model was used to investigate the accuracy of the assumptions made in the SPW TWT black-box model. For example, Figure 1 shows the MAFIA simulated gain as a function of frequency for single tone excitation, with and without reflections. Also shown is the gain for a wideband excitation as would occur in high data rate communications. The discrepancies between the gain ripple behaviors clearly show the inaccuracy of using single-tone excitation to represent a realistic wideband signal.

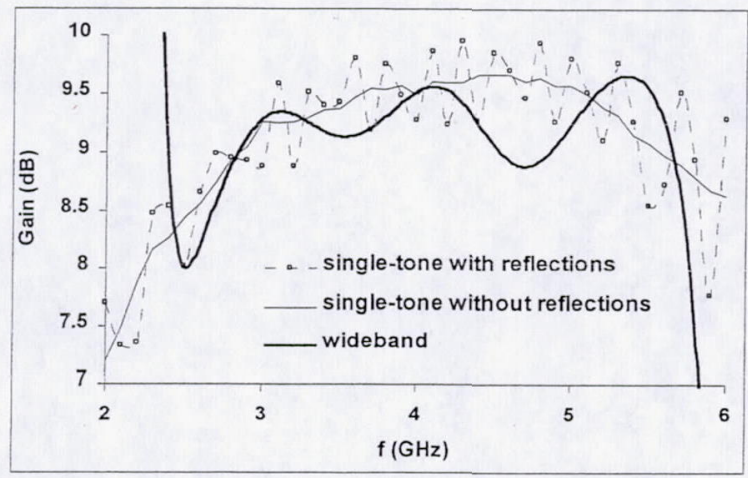

Figure 1 MAFIA simulated gain for single tone and wideband excitation.

In addition, digital signal performance, including ISI, will be compared using direct data input into the MAFIA model and using the system level analysis tool, SPW. Results show significant differences in predicted degradation between SPW and MAFIA simulations, and the differences become more pronounced with larger system bandwidths (See Figure 2) and higher order modulation schemes, such as 64-QAM. These results demonstrate the importance of accurate TWTA models in system level communications simulations, particularly for high-data rate systems, which require large bandwidth and high-order modulation schemes.

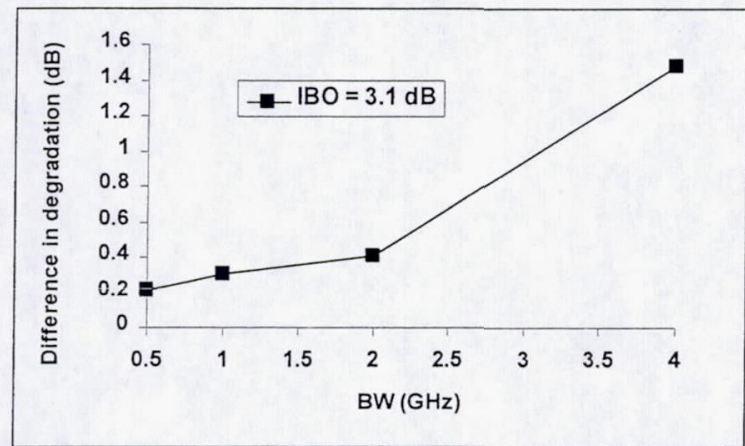

Figure 2 Difference in upper bound degradation between SPW and MAFIA using binary phase shift keying (BPSK) and $3.1 \mathrm{~dB}$ input backoff (IBO) as a function of bandwidth (BW).

\section{Work supported by NASA's Cross Enterprise Technology Development Program in Code R (OAST)}

1. SPW ${ }^{\mathrm{TM}}$, Signal Processing Worksystem. Comdisco Systems, Inc. 919 Hillsdale Blvd., Foster City, CA 94404

2. D. M. MacGregor, Two-dimensional nonlinear multisignal helix traveling-wave tube amplifier computer program, Volume 1:

User Manual, Electrocon International, Inc., Ann Arbor, Michigan, April 1993

3. C. L. Kory, Three-dimensional simulations of PPM Focused Helical Traveling-wave tubes, Doctor of Engineering

Dissertation, Cleveland State University, Cleveland, Ohio, August 2000.

4. MAFIA, Computer Simulation Technology (CST) GmbH (web:www.cst.de)

5. M. C. Jeruchim, P. Balaban, and K. S. Shanmugan, Simulation of Communication Systems. Modeling, Methodology, and

Techniques, $2^{\text {nd }}$ Edition, Kluwer Academic/Plenum Publishers, New York (2000). 